\title{
Beam manipulation with velocity bunching for PWFA applications
}

\author{
R. Pompilia, M.P. Anania ${ }^{a}$, M. Bellaveglia a , A. Biagioni ${ }^{a}$, F. Bisesto ${ }^{a}$, E. Chiadroni ${ }^{a}$, \\ A. Cianchi ${ }^{b}$, M. Croia ${ }^{a}$, A. Curcio ${ }^{a}$, D. Di Giovenale ${ }^{a}$, M. Ferrario ${ }^{a}$, F. Filippi ${ }^{c}$, M. Galletti $^{\text {a }}$, \\ A. Gallo ${ }^{\mathrm{a}}$, A. Giribono ${ }^{\mathrm{c}}$, W. $\mathrm{Li}^{\mathrm{d}}$, A. Marocchino ${ }^{\mathrm{c}}$, A. Mostacci ${ }^{\mathrm{c}}$, M. Petrarca ${ }^{\mathrm{c}}$, V. Petrillo ${ }^{\mathrm{e}}$, \\ G. Di Pirro ${ }^{a}$, S. Romeo ${ }^{\text {a }}$, A.R. Rossi ${ }^{\text {e, J. Scifo }}{ }^{a}$, V. Shpakov ${ }^{a}$, C. Vaccarezza ${ }^{a}$, F. Villa ${ }^{a}$, J. Zhu ${ }^{f}$ \\ a Laboratori Nazionali di Frascati, Via Enrico Fermi 40, 00044 Frascati (Rome), Italy \\ b University of Rome "Tor Vergata" and INFN-Roma Tor Vergata, Via della Ricerca Scientifica 1, 00133 Rome, Italy \\ ' University of Rome "Sapienza", Piazzale Aldo Moro 5, 00185 Rome, Italy \\ d National Synchrotron Radiation Laboratory, University of Science and Technology of China, Hefei, Anhui 230029, China \\ e INFN-Milan and Department of Physics, University of Milan, Via Celoria 16, 20133 Milan, Italy \\ ${ }^{f}$ DESY, Notkestrasse 85, 22603 Hamburg, Germany
}

\section{A R T I C L E I N F O}

Available online 1 February 2016

Keywords:

Velocity bunching

Beam dynamics

Hollow beams

Plasma acceleration

Pwfa

Lwfa

\begin{abstract}
A B S T R A C T
The activity of the SPARC_LAB test-facility (LNF-INFN, Frascati) is currently focused on the development of new plasma-based accelerators. Particle accelerators are used in many fields of science, with applications ranging from particle physics research to advanced radiation sources (e.g. FEL). The demand to accelerate particles to higher and higher energies is currently limited by the effective efficiency in the acceleration process that requires the development of km-size facilities. By increasing the accelerating gradient, the compactness can be improved and costs reduced. Recently, the new technique which attracts main efforts relies on plasma acceleration. In the following, the current status of plasma-based activities at SPARC_LAB is presented. Both laser- and beam-driven schemes will be adopted with the aim to provide an adequate accelerating gradient $(1-10 \mathrm{GV} / \mathrm{m})$ while preserving the brightness of the accelerated beams to the level of conventional photo-injectors. This aspect, in particular, requires the use of ultra-short ( $<100 \mathrm{fs}$ ) electron beams, consisting in one or more bunches. We show, with the support of simulations and experimental results, that such beams can be produced using RF compression by velocity-bunching.
\end{abstract}

(c) 2016 Elsevier B.V. All rights reserved.

\section{Introduction}

Ultra-short electron beams are used in several applications, e.g. Free Electron Lasers (FEL) [1] and as $\mathrm{THz}$ radiation sources [2]. Recently they have been implemented also in novel beam-driven Plasma Wakefield Accelerators (PWFA) [3] and their use is foreseen also in several laser-driven proposals [4,5]. In PWFA, bunches shorter than the plasma wavelength $\lambda_{p}\left(\approx 330 \mu \mathrm{m}\right.$ for $n_{p}=10^{16} \mathrm{~cm}^{-3}$ density) are required, if self-modulation schemes are not considered [6]. Recent advances have been obtained with a driver bunch, exciting the plasma wakefield, followed by a witness gaining the energy released by the driver. The witness has even more stringent requirements: its length must be $\sigma_{z} \ll \lambda_{p}$ in order to avoid energy spread growth. The same parameters apply to Laser Wakefield Acceleration (LWFA) schemes employing the external injection of a witness bunch produced by a photo-injector [7]. Such ultra-short bunches cannot be

E-mail address: riccardo.pompili@lnf.infn.it (R. Pompili). produced directly in a RF gun due to space-charge forces acting within short distances. Instead, it is preferred the production of relatively low peak currents while manipulations on the longitudinal phase space (LPS) are implemented in the downstream transport, e.g. with compression techniques based on RF (velocity-bunching, VB [8]) or magnetic schemes [9]. In the following we focus on the use of VB integrating emittance compensation [10], in view of the planned plasma acceleration experiments at SPARC_LAB.

\section{The SPARC_LAB test-facility}

SPARC_LAB [4] is a test-facility providing electron beams with energies up to $180 \mathrm{MeV}$ feeding four experimental beamlines (Fig. 1). It is based on the combination of high brightness $\left(10^{15} \mathrm{~A} / \mathrm{m}^{2} \mathrm{rad}^{2}\right)$ electron beams from the SPARC photo-injector [11] with high power (200 TW) laser pulses from FLAME [12]. The photo-injector consists of a S-band 1.6 cell BNL/UCLA/SLAC type gun followed by three S-band 


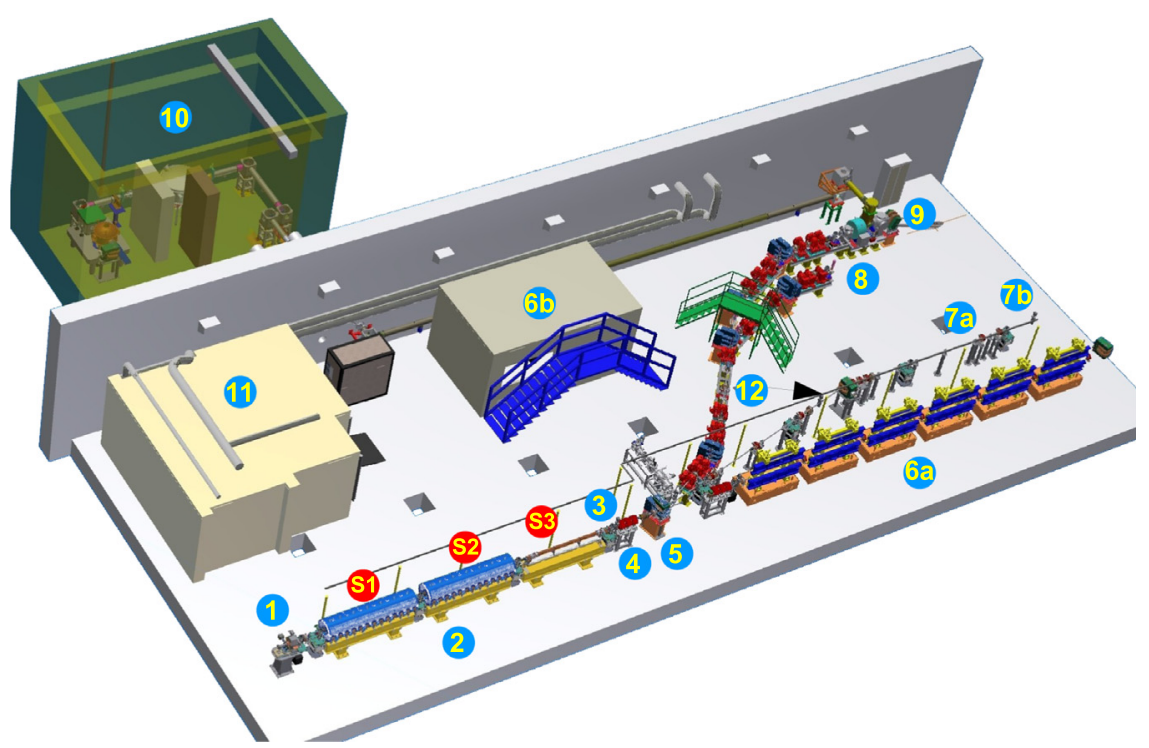

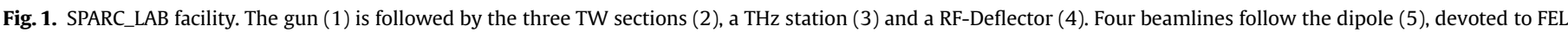

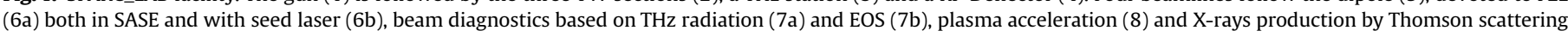
(9) using the FLAME laser (10). EOS shares the laser from the photo-cathode (11) and is delivered by an optical line (12).

TW sections (hereinafter called S1, S2 and S3). Solenoid coils embedding the first two sections provide additional magnetic focusing during the $\mathrm{VB}$ process and take control of emittance oscillations [10]. FLAME is based upon a Ti:Sa chirped pulse amplification (CPA) system delivering $5 \mathrm{~J}$ pulses with $30 \mathrm{fs}$ (FWHM) duration at $800 \mathrm{~nm}$ (fundamental wavelength) and $10 \mathrm{~Hz}$ repetition rate. The laser features a high, sub-ns contrast ratio $\left(>10^{10}\right)$ and can be focused on targets using a $F / 10$ off-axis parabola, resulting in a maximum intensity of $10^{19} \mathrm{~W} / \mathrm{cm}^{2}$. The joint presence of these two systems allows the investigation of several plasma acceleration schemes, e.g. self [13] and external injection [7], laser and beam driven, and a wide spectrum of inter-disciplinary research activity based on radiation sources, e.g. FEL both in SASE, Seeded and exotic schemes [14], X-ray sources by means of Thomson scattering [15] and high power $\mathrm{THz}$ radiation [16]. Studies related to advanced diagnostics demonstrated the feasibility to fully characterize electron beams consisting in one or multiple bunches by using devices based on $\mathrm{THz}$ [17] and Electro-Optical Sampling (EOS) [18] and by extending conventional quadrupole-scan methods [19].

\section{Advanced plasma acceleration activities}

The current SPARC_LAB activity is focused on plasma-based experiments. At the end of 2015 a PWFA experiment will be installed at the linac exit, upstream the FEL beamline. First attempts of plasma acceleration are planned in 2016. In addition to the beamdriven approach, a dedicated beamline is dedicated for a LWFA experiment driven by FLAME and employing the external injection of a witness bunch from SPARC.

\subsection{Plasma acceleration by PWFA}

There are several possible PWFA scenarios when operating such experiments, mainly depending on the working regime (linear or nonlinear). The linear limit has the advantage that the plasma oscillation may be resonantly driven, but has the notable drawback that mobile plasma electrons exist inside of the beam channel, which in turn implies that focusing is nonlinear in radial coordinate $r$ and timedependent; the acceleration would be dependent on $r$, too. These drawbacks can be overcome with the so-called blow-out regime [20]: if the beam density is much greater than the plasma density $\left(n_{b} \gg n_{p}\right)$, the beam channel is totally rarefied of plasma electrons. Under these conditions the plasma response is very nonlinear, with relativistic electron velocities achieved, an amplitude-dependent plasma wavelength $\lambda_{p}$ and large wave-breaking spikes at the end of the first oscillation. To overcome these limitations, the beam-driven experiment at SPARC_LAB will operate in the so-called quasi-nonlinear $(\mathrm{QNL})$ regime [21]. Being $N_{b}$ the driver number of particles, $k_{p}=2 \pi / \lambda_{p}$ and $r_{e}$ the classical electron radius, the key parameter to measure the non-linearity of the plasma response is identified by the dimensionless charge quantity:

$\tilde{Q} \equiv \frac{N_{b} k_{p}^{3}}{n_{p}}=4 \pi k_{p} r_{e} N_{b}$

If $\tilde{Q} \ll 1$ ( $>1$ ) the regime is linear (nonlinear). A QNL regime is given by the condition $\tilde{Q}<1$ for linearity combined to $n_{b} / n_{p}>1$ for bubble formation, with $n_{b}$ the bunch charge density. Since optimal conditions for a linear response is based on $k_{p} \sigma_{z} \approx \sqrt{2}$, a QNL regime requires very thin beams, $\left(\sigma_{r} \ll \sigma_{z}\right.$, with $\sigma_{r}$ the bunch transverse size). Being the wave-breaking field $E_{W B}(V / m) \approx 96 \sqrt{n_{p}\left(\mathrm{~cm}^{-3}\right)}$, the accelerating field is [22]

$E_{z} \approx 1.3 E_{W B} \frac{n_{b}}{n_{p}} k_{p}^{2} \sigma_{r}^{2} \log \left(\frac{1}{k_{p} \sigma_{r}}\right)$.

Since $\lambda_{p}$ is amplitude independent, the QNL regime can be used also in resonant schemes with multiple drivers separated in time by $\lambda_{p}$ [23]. It allows to produce an accelerating field proportional to the number of drivers while handling bunches with lower charges and emittances. At SPARC_LAB, a 3-5 cm-long sapphire capillary (1 mm diameter) filled by Hydrogen gas will be implemented. A HV discharge circuit $(20 \mathrm{kV}$, $200 \mathrm{~A}$ ) is used to ionize the gas and provide $n_{p} \approx 10^{16} \mathrm{~cm}^{-3}$ plasma densities, corresponding to $\lambda_{p} \approx 330 \mu \mathrm{m}$ and an oscillation period $T_{p} \approx 1 \mathrm{ps}$. The goal of the beam-driven experiment is to provide an adequate accelerating gradient of the order of $1 \mathrm{GV} / \mathrm{m}$ while preserving the witness quality, i.e. low energy spread $(\Delta E / E<1 \%)$ and emittance ( $\epsilon_{n} \lesssim 1 \mathrm{~mm}$ mrad).

\subsection{Plasma acceleration by external injection in LWFA}

A new scheme based on LWFA that combines the use of the highpower FLAME laser and the high-brightness SPARC photo-injector will be realized at SPARC_LAB. It exploits the plasma wake excitation using 
an ultra-short laser pulse followed by the injection of a witness bunch coming from the photo-injector. Since self-injection from plasma is prevented, we aim to combine large accelerating gradients with high quality, stable and reproducible beams typical of conventional photoinjectors [24]. With this purpose an ultra-short $(\approx 35 \mathrm{fs}$, rms) high energy ( $3.5 \mathrm{~J}$ ) laser pulse coming from FLAME will be used in order to produce plasma ( $n_{p} \approx 10^{17} \mathrm{~cm}^{-3}$ ) and excite the wakefield. By considering that the normalized wave potential is in the range $1 \leq a_{0} \leq 1.3$, the working regime is mildly nonlinear and the accelerating field scales as [25]

$E_{z} \approx \frac{a_{0}^{2}}{\sqrt{1+a_{0}^{2}}} E_{W B}$.

The plasma will be confined in a thin capillary (100 $\mu$ m diameter), acting as an optical wave-guide to propagate the laser over distances longer than the Rayleigh range [26].

\section{Velocity-bunching with emittance compensation}

Ultra-short bunches, as the one needed in PWFA and LWFA, can be obtained with VB. It requires the injection in the first TW section (S1) at the zero-crossing of the RF wave in order to longitudinally compress the bunch by accelerating (decelerating) its tail (head) [8,27]. Fig. 2 reports the longitudinal phase-space of a $400 \mathrm{pC}$ bunch for several injection phases in S1. For the simulation we used the General Particle Tracer (GPT) code [28]. The VB process is based on a correlated beam time-velocity chirp, i.e. electrons on the tail of the bunch are faster than the ones in the head. This leads to a rotation of the LPS if the injected beam is slower than the phase velocity of the RF wave so that when injected at the zero-crossing field phase it slips back to phases where the field is accelerating, but is simultaneously chirped and compressed. By changing the injection phase, the beam is shortened until a maximum compression point $\left(\phi_{S 1}=-89^{\circ}\right)$ corresponding to a duration $\sigma_{t} \approx 60 \mathrm{fs}$ and about $2.1 \mathrm{kA}$ peak current. In order to avoid an excessive emittance growth, the S1 embedded solenoids are turned on to provide the necessary additional focusing, as required by the compensation process of emittance [10], whose resulting value is $\epsilon_{n} \approx 2.2 \mathrm{~mm}$ mrad.

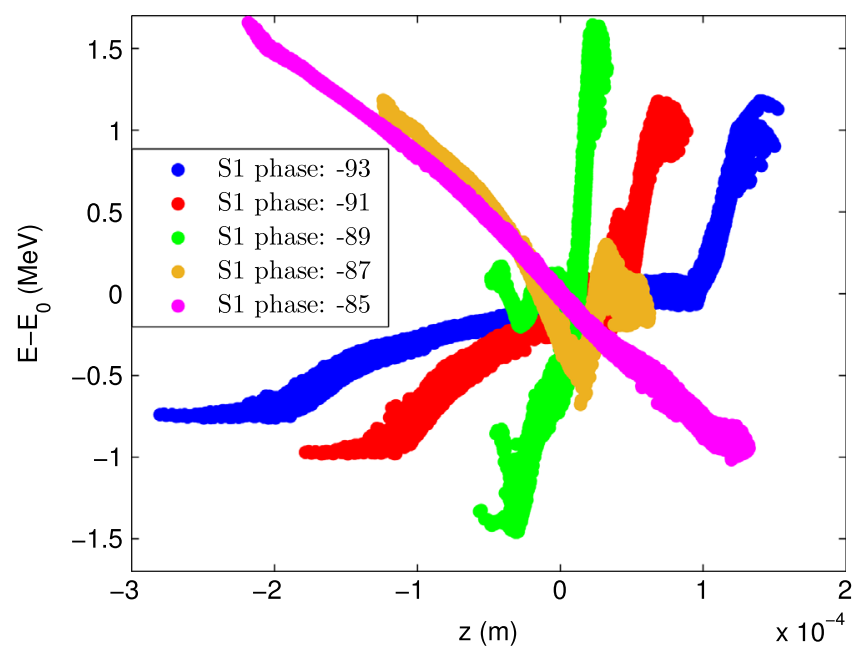

Fig. 2. $400 \mathrm{pC}$ beam LPS at linac exit. Several S1 injection phases are reported, highlighting the LPS rotation during the compression process.
Table 1

Laser and electron beam parameters at linac exit $(z=12 \mathrm{~m})$.

\begin{tabular}{lll}
\hline Photo-cathode laser & Driver & Witness \\
\hline $\begin{array}{l}\text { Spot @ cathode (mm) } \\
\text { Duration (fs) }\end{array}$ & 1.3 & 0.6 \\
& 100 & 100 \\
Electron bunch & Driver & Witness \\
\hline Charge (pC) & 200 & 20 \\
Duration (fs) & 190 & 40 \\
Energy (MeV) & 107 & 107 \\
Emittance (mm mrad) & 3.9 & 2.7 \\
D-W distance (ps) & 0.55 & \\
\hline
\end{tabular}

\section{Electron bunches for PWFA}

In PWFA a charge-density wake is driven by the passage of an ultra-relativistic bunch of charged particles (the driver) through a plasma. In previous experiments the accelerated charge arose from the driver itself [29]; more recently, a short witness bunch has been accelerated [3] in order to preserve its properties and avoid an excessive energy spread and emittance growth. At SPARC_LAB, a technique called laser-comb is routinely used in order to produce trains of multiple electron bunches [30]. In this operating mode, the photo-cathode is illuminated by a comb-like laser pulse to extract a train of electron bunches that are injected into the same RF bucket [31]. With respect to other methods [32], this one is able to preserve all extracted charge. In the following, start-to-end simulations are provided with the aim to test the PWFA scheme and its feasibility at SPARC_LAB. Experimental results (even with multiple-bunches beams) are reported, too.

\subsection{Photo-injector setup}

The use of fs-laser pulses in high-gradient RF guns enables the production of bunches whose length is much smaller than the radius ("pancake" regime). Being $E_{0}=E_{\max } \sin \phi_{0}$, where $E_{\max }$ is the peak field at cathode and $\phi_{0}$ the injection phase, the pancake bunch during acceleration will evolve into an uniformly filled 3D ellipsoid [33] if it meets the condition

$\frac{e E_{0} \tau_{L}}{m_{e} c} \ll \frac{\sigma_{0}}{\epsilon_{0} E_{0}} \ll 1$

where $\tau_{L}$ is the laser pulse duration and $\sigma_{0}$ is the surface charge density. The uniformly filled 3D ellipsoid represents a highly idealized distribution because of its linear internal force fields. Being $Q$ the total extracted charge and $r_{L}$ the (rms) laser radius, the second term in Eq. (4) is essentially the ratio between $E_{\text {image }}=\sigma_{0} / 2 \epsilon_{0}=Q / 2 \pi r_{L}^{2} \epsilon_{0}$, the electric field due to image charge on the cathode, and $E_{0}$. It has been demonstrated that emittance decreases by lowering such ratio [34], i.e. with the bunch approaching the uniform ellipsoidal shape.

A potential PWFA scheme consisting in a $200 \mathrm{pC}$ driver followed by a $20 \mathrm{pC}$ witness is summarized in Table 1 . The initial laser configuration is chosen in order to fulfill Eq. (4). The simulation has been obtained by using two laser pulses separated by $2.4 \mathrm{ps}$. Since during the VB process the LPS is rotated, the position of bunches is reversed at the end of the process. ${ }^{1}$ The resulting distance is $550 \mathrm{fs}$, corresponding to $\lambda_{p} / 2$ (for $n_{p}=10^{16} \mathrm{~cm}^{-3}$ ), i.e. the accelerating and focusing region in the plasma bubble. From the reported values it is $n_{b} \approx 5 n_{p}$, resulting in $\tilde{Q} \approx 0.8$, as required by the QNL regime. The bunch duration and emittance evolution is shown in Fig. 3.

\footnotetext{
${ }^{1}$ It means that in order to have the witness following the driver at the linac exit, the witness must be released before the driver from the cathode.
} 


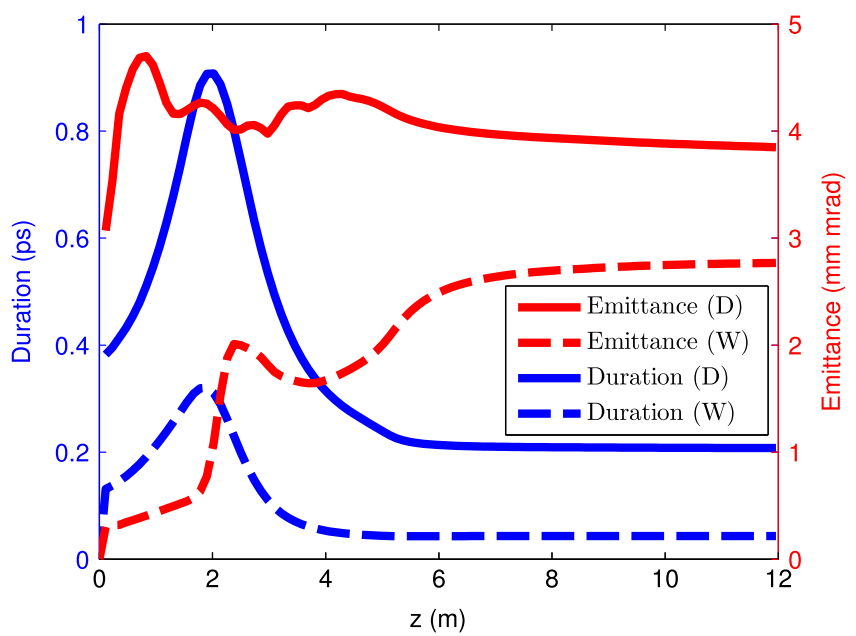

Fig. 3. Driver (solid) and witness (dashed) evolution along the SPARC_LAB photoinjector. The blue (red) lines refer to the bunch duration (emittance). (For interpretation of the references to color in this figure caption, the reader is referred to the web version of this paper.)

\subsection{PWFA simulations}

For the successful operation of a PWFA the beam must be transversely matched to the plasma in terms of the Twiss $\beta$-function. Being $\gamma$ the relativistic Lorentz factor,

$\beta_{e q}=\sqrt{\frac{\gamma}{2 \pi r_{e} n_{p}}}$.

represents the matching condition [35]. With $n_{p}=10^{16} \mathrm{~cm}^{-3}$, it results $\beta_{e q} \approx 1.1 \mathrm{~mm}$. For this purpose a triplet of permanent magnet quadrupoles (PMQ) has been specifically designed in order to provide $520 \mathrm{~T} / \mathrm{m}$ gradient. They consist in 12 magnetized sectors $\left(B_{r} \approx 1.3 \mathrm{~T}\right)$ arranged according to the Halbach geometry [36]. The PMQs ( $f \approx 14 \mathrm{~cm}$ ) allow to focus the driver (witness) to a final spot of $\sigma_{x}=5.5(4) \mu \mathrm{m}$.

The PWFA simulation is performed with a new hybrid-kinetic fluid code called Architect [37] that works as a PIC code for the electron bunches while treating the plasma as a fluid. Fig. 4(a) shows the energies (blue line) of the two bunches while propagating in a $3 \mathrm{~cm}$ plasma channel as resulting from the simulation. The witness energy spread (red line) is reported, too. The results point out that the witness experienced about $1.1 \mathrm{GV} / \mathrm{m}$ accelerating fields and, thanks to its longitudinal matching and short duration, the cumulated energy spread is quite low $(\Delta E / E \approx 1.5 \%)$. The witness envelope and emittance are shown in Fig. 4(b). The resulting matching shows that envelope oscillations are mitigated and emittance is almost constant.

\subsection{Hollow driver bunches}

In Section 5.2 the witness duration and emittance are, respectively, $40 \mathrm{fs}$ (rms) and $2.7 \mathrm{~mm}$ mrad (cf. Table 1). As shown in Fig. 3, the emittance starts increasing from $z \approx 4 \mathrm{~m}$, the point corresponding to the crossover of the two bunches, i.e. when they are overlapped in time. Emittance growth is due to space-charge effects that, when considering two crossing beams with the same particles, act as a nonlinear diverging lens. With a proper shaping of the driver transverse profile the effects can be reduced. A hollow beam can be exploited in order to provide lower charge density in the core [38], allowing the witness to pass through it without feeling any nonlinear defocusing field. Such profile can be obtained by implementing an hollow laser pulse on the cathode, with a proper choice of inner and outer radii. The transverse electric field (due to space-charge) for a

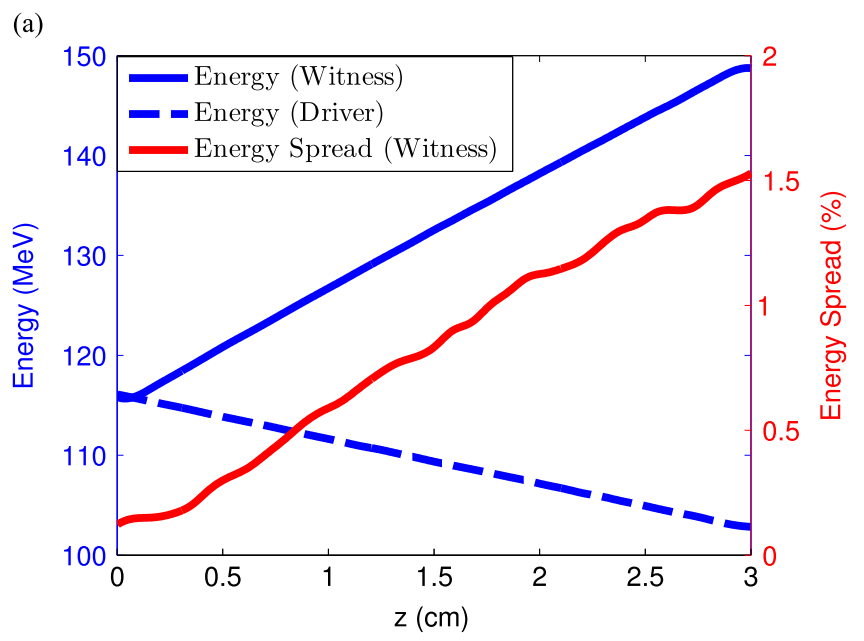

(b)

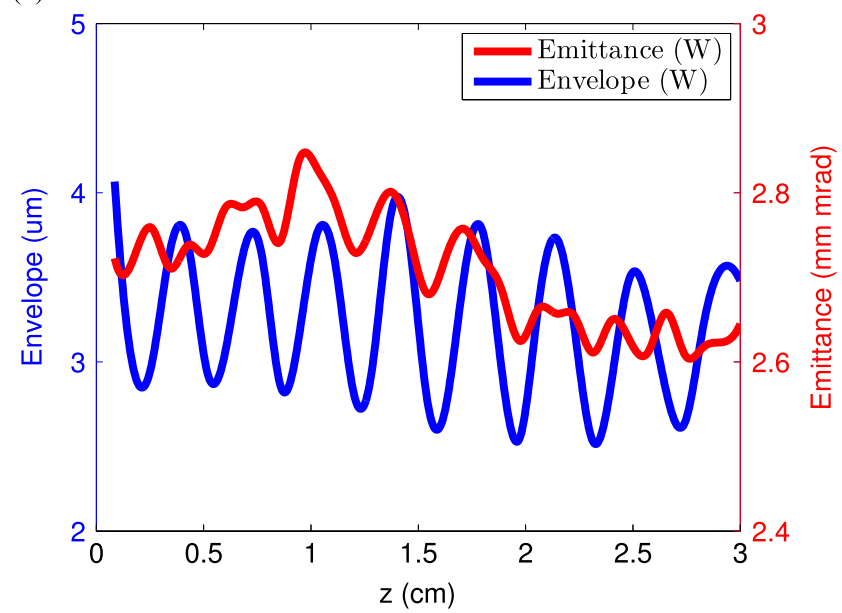

Fig. 4. Beam evolution along the capillary. (a) Energy gain (blue) for witness (solid) and driver (dashed); the witness energy spread (red) is reported, too. In (b) the envelope (blue) and emittance (red) of the witness bunch are reported. (For interpretation of the references to color in this figure caption, the reader is referred to the web version of this paper.)

hollow beam centered in $r_{0}$ and having width $\sigma_{r}$ is given by

$E_{r}(r)=\frac{n_{b}}{\epsilon_{0}} \frac{\sigma_{r}^{2}}{r}\left(\mathrm{e}^{-r_{0}^{2} / 2 \sigma_{r}^{2}}-\mathrm{e}^{-\left(r-r_{0}\right)^{2} / 2 \sigma_{r}^{2}}\right)$.

For pure Gaussian beams $\left(r_{0}=0\right)$ it is $E_{r}>0$, i.e. particles are pushed outwards. Instead, in hollow beams $\left(r_{0}>0\right)$ it is $E_{r}<0$ for $r<r_{0}$ meaning that space-charge forces are directed inwards, i.e. particles move towards the axis. In order to avoid the hole filling, $r_{0}$ must be large enough. If the hole is completely charge-free, then $E_{r}=0$. Table 2 summarizes the simulation results. For witness it is $\epsilon_{n} \approx 0.4 \mathrm{~mm}$ mrad, about seven times lower (cf. Table 1). Fig. 5 shows the bunch crossing during VB. The driver (blue) hole is large enough to allow the witness (red) crossing. This configuration proves that witness quality can be preserved even in VB with multiple-bunches.

\subsection{Recent results at SPARC_LAB}

As described in Section 5.2, a first scheme of comb-like beam consists in a driver $(200 \mathrm{pC})$ followed by a witness $(20 \mathrm{pC})$. In order to provide a fine tuning of the witness position with respect to the driver, the witness laser pulse can be delayed or anticipated with a delay line. Fig. 6 (a)-(c) shows the experimental LPS. While the driver duration is fixed in VB by the S1 injection phase, the witness position and duration can be tuned with the delay line. In this case a range of \pm 1 ps has been explored; in Fig. 6 the two bunches overlapped in 
Table 2

Parameters for the hollow driver configuration at linac exit $(z=12 \mathrm{~m})$.

\begin{tabular}{lll}
\hline Laser pulse & Driver & Witness \\
\hline Spot @ cathode (mm) & $3.4\left(r_{0}\right), 0.9\left(\sigma_{r}\right)$ & 0.55 \\
Duration (fs) & 100 & 100 \\
Electron bunch & Driver & Witness \\
\hline Charge (pC) & 200 & 20 \\
Duration (fs) & 145 & 29 \\
Energy (MeV) & 107 & 107 \\
Emittance (mm mrad) & 6.2 & 0.4 \\
D-W Distance (ps) & 0.55 & \\
\hline
\end{tabular}

(a)

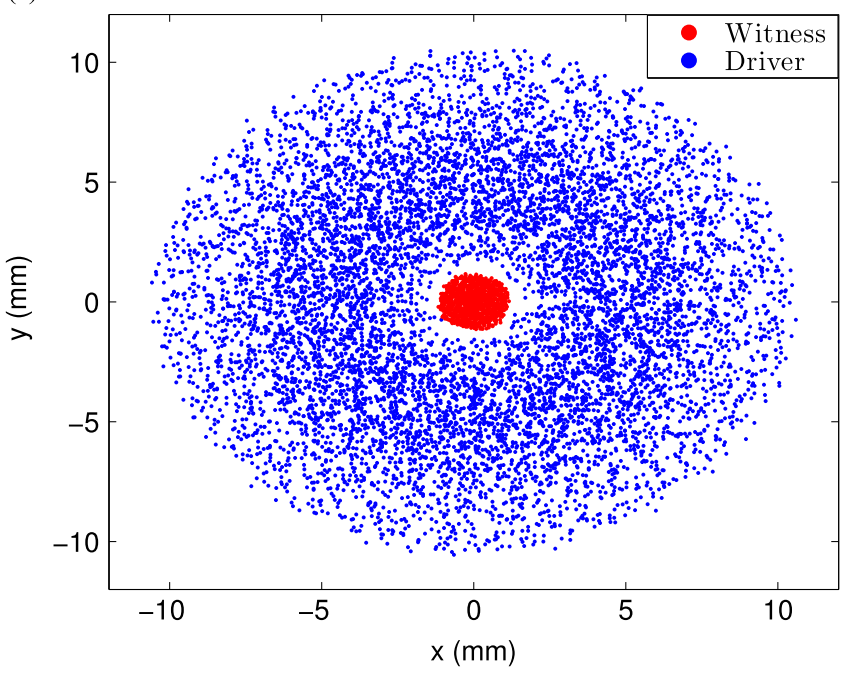

(b)

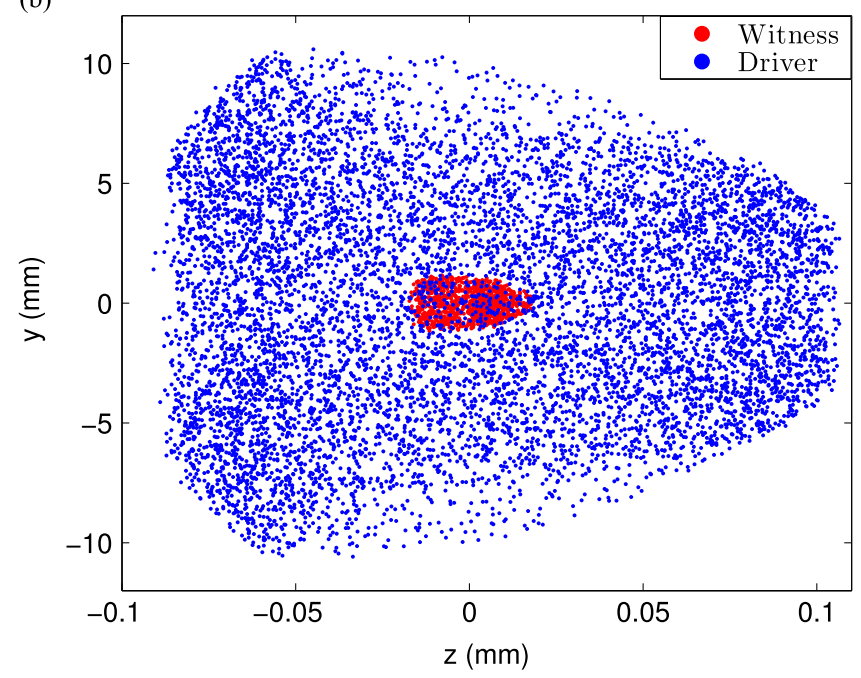

Fig. 5. Transverse (a) and longitudinal (b) view of driver-witness crossing.

time. Fig. 6 (d)-(f) shows several comb-like beams consisting in multiple drivers tested at SPARC_LAB. The reported LPS consist in two (Fig. 6(d)) and four (Fig. 6(e)) $50 \mathrm{pC}$ drivers followed by a $20 \mathrm{pC}$ witness. Fig. $6(\mathrm{f})$, in particular, shows the four drivers with a charge ramp $(15,35,60$ and $90 \mathrm{pC})$. Being about one $\lambda_{p}(\approx 1 \mathrm{ps})$ the separation between drivers, these results represent a viable configuration for resonant plasma acceleration schemes.

\section{External injection in LWFA}

External injection in LWFA has many technical difficulties which have to be overcome, e.g. ultra-low relative timing-jitters between FLAME laser and electron bunches [7]. In the following, several results are presented. The aim is to be able to accomplish the requirements reported in Table 3. Section 6.1 shows recent results, in particular regarding the timing-jitter reduction.

\subsection{Recent results at SPARC_LAB}

The most demanding requirement is to provide a fs-level synchronization between the pump laser producing the plasma wake and the externally injected ultra-short bunch, i.e. the bunch arrival timingjitter (ATJ) with respect to the laser must be kept as low as possible. In order to provide ultra-short bunches a compressor, either RF (VB) or magnetic, is needed. It follows that the beam arrival-time is tied to compressor jitters and uncoupled from the photo-cathode (PC) laser timing [39]. As a result the ATJ relative to the PC laser results increased downstream the compressor. We proved that, by means of a new hybrid compression scheme consisting in the combined use of $\mathrm{VB}$ and magnetic compression (MC) in a dogleg, it is possible to obtain ultrashort bunches with ultra-low relative ATJ. The underlying principle relies on space-charge forces that strongly alter the bunch longitudinal phase space while leaving almost unperturbed its mean energy and time-of-flight. PC laser arrival time and RF accelerating field jitters have therefore different impacts on the bunch longitudinal dynamics (with space-charge) with respect to its shot-to-shot longitudinal centroids distribution (space-charge free). It follows that the time-energy correlation (i.e. the chirp term) is different in the two cases, thus a dispersive beamline provides different longitudinal compressions for the bunch and its time-of-arrival [40]. In the hybrid scheme the bunch is initially compressed by VB, resulting in an increased timing-jitter relative to the PC laser. Nevertheless, the shotto-shot longitudinal centroids distribution has a different time-energy distribution with respect to the bunch one and the dogleg can be exploited to restore (i.e. compress) the timing-jitter relative to the PC laser while slightly elongating the VB compressed bunch. We exploited such hybrid scheme achieving a relative timing-jitter of $19 \mathrm{fs}$ (rms). This measurement has been obtained with an Electro-Optical Sampling (EOS) device employing a probe laser directly split from the PC laser system [18]. All results are summarized in Table 3.

\section{Conclusions}

Current activities performed at the SPARC_LAB facility have been presented in view of LWFA and PWFA experiments. The aim is to prove high accelerating gradients in plasmas and, at the same time, preserve the high brightness of electron bunches coming from current photo-injectors. In the first case, more emphasis is put on the very demanding precondition to have ultra-low timing-jitters between the pump laser and the injected beam. In the second one, beams consisting in several sub-bunches can be handled and shaped in order to fully accomplish the requirements for injection (bunch length, spacing, charge, energy and emittance). For the PWFA, simulation results reported in Section 5.3 demonstrate that, when considering multiple-bunches schemes, hollow drivers allow to preserve the witness quality, avoiding emittance growth during velocity-bunching. It represents a possible approach for PWFA in order to reach current photoinjector performances in terms of brightness. 
(a)

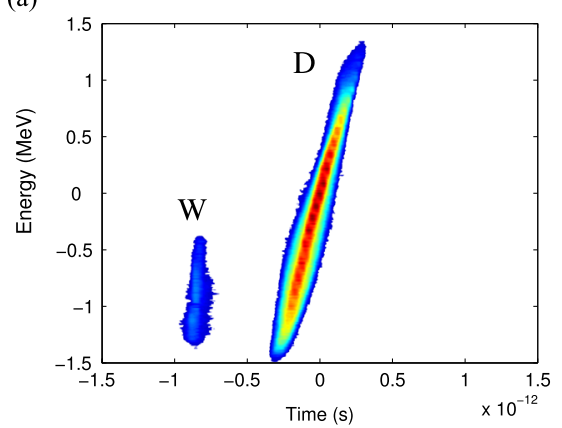

(d)

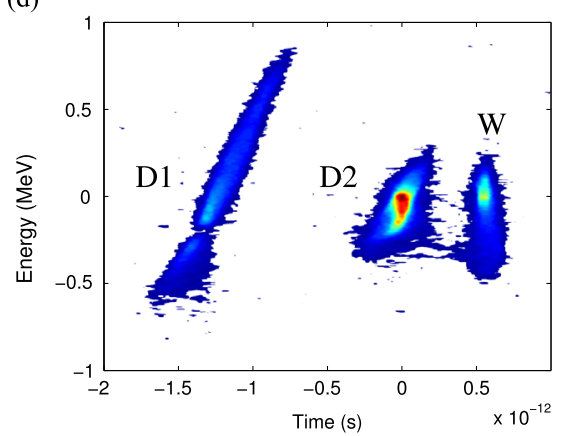

(b)

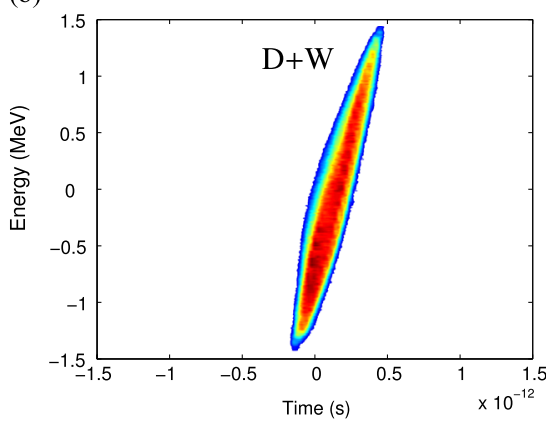

(e)

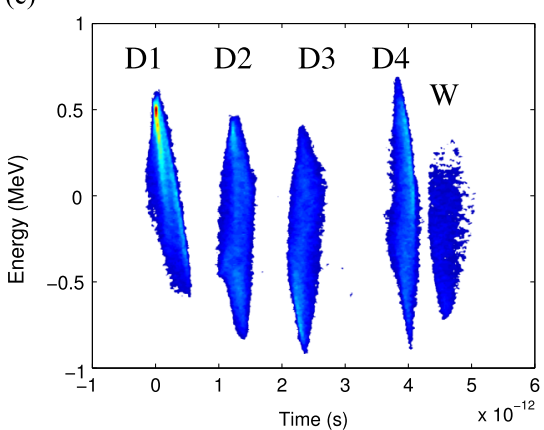

(c)

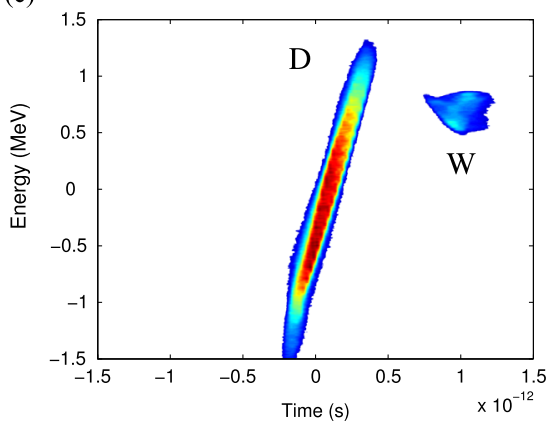

(f)

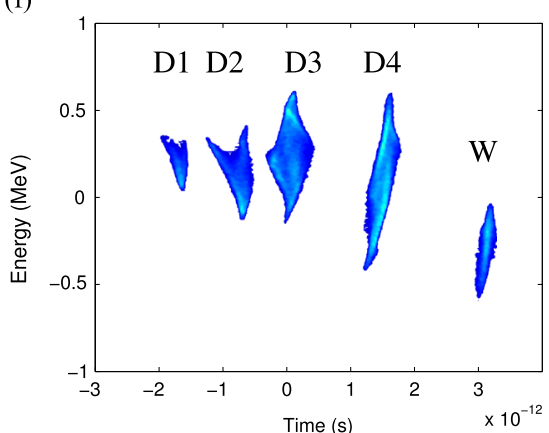

Fig. 6. Experimental data. (a)-(c) LPS of a comb-like beam consisting in a driver (D, $200 \mathrm{pC}, 130 \mathrm{fs})$ and a witness (W, $20 \mathrm{pC}, 90 \mathrm{fs})$. Each LPS is obtained by tuning the initial delay of the witness. (d)-(f) Multi-driver ( $50 \mathrm{pC}$ ) configurations. The driver durations are 80 and $70 \mathrm{fs}$ (d); 300, 90, 90 and $80 \mathrm{fs}$ (e)-(f). The witness bunch (20 pC, $50 \mathrm{fs}$ ) is on the right in all plots. In (f) the drivers have a charge ramp $(15,35,60$ and $90 \mathrm{pC})$. The reported durations are rms.

Table 3

Requirements vs. current status for external-injection in LWFA.

\begin{tabular}{lll}
\hline Parameter & Requirements & Experiments \\
\hline Charge (pC) & 10 & 20 \\
Duration (fs, rms) & 17 & 26 \\
Energy (MeV) & 80 & 80 \\
Emittance (mm mrad) & 1.0 & 1.2 \\
Timing-jitter (fs) & $<30^{\mathrm{a}}$ & $19^{\mathrm{b}}$ \\
\hline
\end{tabular}

a The timing-jitter is relative to the pump laser.

${ }^{b}$ The timing-jitter is relative to the photo-cathode laser.

\section{Acknowledgments}

This work has been partially supported by the EU Commission in the Seventh Framework Program, Grant Agreement 312453-EuCARD2 and the Italian Research Minister in the framework of FIRB - Fondo per gli Investimenti della Ricerca di Base, Project n. RBFR12NK5K.

\section{References}

[1] L. Giannessi, A. Bacci, M. Bellaveglia, F. Briquez, M. Castellano, E Chiadroni, A. Cianchi, F. Ciocci, M. Couprie, L. Cultrera, et al., Physical Review Letters 106 (14) (2011) 144801.

[2] N. Stojanovic, M. Drescher, Journal of Physics B: Atomic, Molecular and Optical Physics 46 (19) (2013) 192001.

[3] M. Litos, E. Adli, W. An, C. Clarke, C. Clayton, S. Corde, J. Delahaye, R. England, A. Fisher, J. Frederico, et al., Nature 515 (7525) (2014) 92.

[4] M. Ferrario, D. Alesini, M. Anania, A. Bacci, M. Bellaveglia, O. Bogdanov, R. Boni, M. Castellano, E. Chiadroni, A. Cianchi, et al., Nuclear Instruments and Methods in Physics Research Section B: Beam Interactions with Materials and Atoms 309 (2013) 183.

[5] R. Assmann, C. Behrens, R. Brinkmann, U. Dorda, K. Flöttmann, B. Foster, J. Grebenyuk, M. Gross, I. Hartl, M. Hüning, et al., Sinbad-a proposal for a dedicated accelerator research facility at desy, in: Proceedings of IPAC2014, Dresden, Germany, TUPME047, 2014, 〈http://accelconf.web.cern.ch/AccelConf/ IPAC2014/papers/tupme047.pdf).
[6] R. Assmann, R. Bingham, T. Bohl, C. Bracco, B. Buttenschön, A. Butterworth, A. Caldwell, S. Chattopadhyay, S. Cipiccia, E. Feldbaumer, et al., Plasma Physics and Controlled Fusion 56 (8) (2014) 084013.

[7] A.R. Rossi, A. Bacci, M. Belleveglia, E. Chiadroni, A. Cianchi, G. Di Pirro, M. Ferrario, A. Gallo, G. Gatti, C. Maroli, et al., Nuclear Instruments and Methods in Physics Research Section A: Accelerators, Spectrometers Detectors and Associated Equipment 740 (2014) 60.

[8] L. Serafini, M. Ferrario, Velocity bunching in photo-injectors, in: American Institute of Physics Conference Series, vol. 581, 2001, pp. 87-106.

[9] P. Piot, State of Art Electron Bunch Compression, Technical Report, 2004.

[10] M. Ferrario, D. Alesini, A. Bacci, M. Bellaveglia, R. Boni, M. Boscolo, M. Castellano, E. Chiadroni, A. Cianchi, L. Cultrera, et al., Physical Review Letters 104 (5) (2010) 054801.

[11] D. Alesini, et al., Nuclear Instruments and Methods in Physics Research Section A: Accelerators, Spectrometers Detectors and Associated Equipment 507 (1-2) (2003) 345, http://dx.doi.org/10.1016/S0168-9002(03)00943-4 〈http://www. sciencedirect.com/science/article/pii/S0168900203009434).

[12] L. Gizzi, M. Anania, G. Gatti, D. Giulietti, G. Grittani, M. Kando, M. Krus, L. Labate, T. Levato, Y. Oishi, et al., Nuclear Instruments and Methods in Physics Research Section B: Beam Interactions with Materials and Atoms 309 (2013) 202.

[13] L. Gizzi, F. Anelli, C. Benedetti, C. Cecchetti, A. Clozza, G. Di Pirro, N. Drenska, R. Faccini, D. Giulietti, D. Filippetto, et al., Il Nuovo cimento della Società italiana di fisica C 32 (3) (2009) 433.

[14] V. Petrillo, M. Anania, M. Artioli, A. Bacci, M. Bellaveglia, E. Chiadroni, A. Cianchi, F. Ciocci, G. Dattoli, D. Di Giovenale, et al., Physical Review Letters 111 (11) (2013) 114802.

[15] A. Bacci, D. Alesini, P. Antici, M. Bellaveglia, R. Boni, E. Chiadroni, A. Cianchi C. Curatolo, G. Di Pirro, A. Esposito, et al., Journal of Applied Physics 113 (19) (2013) 194508.

[16] E. Chiadroni, M. Bellaveglia, P. Calvani, M. Castellano, L. Catani, A. Cianchi, G. D. Pirro, M. Ferrario, G. Gatti, O. Limaj, S. Lupi, B. Marchetti, A. Mostacci, E. Pace, L. Palumbo, C. Ronsivalle, R. Pompili, C. Vaccarezza, Review of Scientific Instruments 84 (2) (2013) 022703, http://dx.doi.org/10.1063/1.4790429 〈http://link.aip.org/link/?RSI/84/022703/1〉.

[17] E. Chiadroni, A. Bacci, M. Bellaveglia, M. Boscolo, M. Castellano, L. Cultrera G. Di Pirro, M. Ferrario, L. Ficcadenti, D. Filippetto, et al., Applied Physics Letters 102 (2013) 094101.

[18] R. Pompili, A. Cianchi, D. Alesini, M. Anania, A. Bacci, M. Bellaveglia M. Castellano, E. Chiadroni, D. Di Giovenale, G. Di Pirro, et al., Nuclear Instruments and Methods in Physics Research Section A: Accelerators, Spectrometers, Detectors and Associated Equipment 740 (2014) 216.

[19] A. Cianchi, D. Alesini, M. Anania, A. Bacci, M. Bellaveglia, M. Castellano, E. Chiadroni, D. Di Giovenale, G. Di Pirro, M. Ferrario, et al., Physical Review Special Topics-Accelerators and Beams 18 (8) (2015) 082804.

[20] J. Rosenzweig, B. Breizman, T. Katsouleas, J. Su, Physical Review A 44 (10) (1991) R6189. 
[21] J. Rosenzweig, G. Andonian, S. Barber, M. Ferrario, P. Muggli, B. O'Shea, Y. Sakai, A. Valloni, O. Williams, Y. Xi, et al., Plasma wakefields in the quasi-nonlinear regime: experiments at atf, in: Advanced Accelerator Concepts: 15th Advanced Accelerator Concepts Workshop, vol. 1507, AIP Publishing, Austin, 2012, pp. 612-617.

[22] W. Lu, C. Huang, M. Zhou, W. Mori, T. Katsouleas, Physics of Plasmas 12 (2005) 063101.

[23] E. Kallos, P. Muggli, I. Pavlishin, I. Pogorelsky, D. Stolyarov, V. Yakimenko, et al., Plasma wakefield acceleration utilizing multiple electron bunches, in: 2007 IEEE Particle Accelerator Conference, PAC, IEEE, Albuquerque, 2007, pp. 30703072.

[24] A. Rossi, et al., Nuclear Instruments and Methods in Physics Research Section A 829 (2016) 67.

[25] E. Esarey, C.B. Schroeder, W.P. Leemans, Reviews of Modern Physics 81 (2009) 1229, http://dx.doi.org/10.1103/RevModPhys.81.1229.

[26] B. Cros, C. Courtois, G. Matthieussent, A. Di Bernardo, D. Batani, N. Andreev, S. Kuznetsov, Physical Review E 65 (2) (2002) 026405.

[27] S. Anderson, P. Musumeci, J. Rosenzweig, W. Brown, R. England, M. Ferrario J. Jacob, M. Thompson, G. Travish, A. Tremaine, et al., Physical Review Special Topics-Accelerators and Beams 8 (1) (2005) 014401.

[28] M. De Loos, S. Van der Geer, General particle tracer: a new 3d code for accelerator and beamline design, in: 5th European Particle Accelerator Conference, 1996, p. 1241.

[29] I. Blumenfeld, C.E. Clayton, F.J. Decker, M.J. Hogan, C. Huang, R. Ischebeck, R. Iverson, C. Joshi, T. Katsouleas, N. Kirby, W. Lu, K.A. Marsh, W.B. Mori, P. Muggli, E. Oz, R.H. Siemann, D. Walz, M. Zhou, Nature 445 (2007) 741, http: //dx.doi.org/10.1038/nature05538.

[30] M. Ferrario, D. Alesini, A. Bacci, M. Bellaveglia, R. Boni, M. Boscolo, P. Calvani, M. Castellano, E. Chiadroni, A. Cianchi, L. Cultrera, G. di Pirro, L. Ficcadenti,
D. Filippetto, A. Gallo, G. Gatti, L. Giannessi, M. Labat, S. Lupi, B. Marchetti, C. Marrelli, M. Migliorati, A. Mostacci, D. Nicoletti, E. Pace, L. Palumbo, V. Petrillo, M. Quattromini, C. Ronsivalle, A.R. Rossi, J. Rosenzweig, L. Serafini, M. Serluca, B. Spataro, H. Tomizawa, C. Vaccarezza, C. Vicario, Nuclear Instruments and Methods in Physics Research Section A 637 (2011) 43, http: //dx.doi.org/10.1016/j.nima.2010.02.018.

[31] F. Villa, S. Cialdi, M. Anania, G. Gatti, F. Giorgianni, R. Pompili, Nuclear Instruments and Methods in Physics Research Section A: Accelerators, Spectrometers, Detectors and Associated Equipment 740 (2014) 188.

[32] M. Hogan, T. Raubenheimer, A. Seryi, P. Muggli, T. Katsouleas, C. Huang, W. Lu, W. An, K. Marsh, W. Mori, et al., New Journal of Physics 12 (5) (2010) 055030.

[33] O. Luiten, S. Van der Geer, M. De Loos, F. Kiewiet, M. Van Der Wiel, Physical Review Letters 93 (9) (2004) 094802.

[34] P. Musumeci, J. Moody, R. England, J. Rosenzweig, T. Tran, Physical Review Letters 100 (24) (2008) 244801.

[35] N. Barov, J.B. Rosenzweig, Physical Review E 49 (5) (1994) 4407.

[36] K. Halbach, Nuclear Instruments and Methods in Physics Research Section A 169 (1) (1980) 1.

[37] A. Marocchino, et al., Nuclear Instruments and Methods in Physics Research Section A 829 (2016) 386, http://dx.doi.org/10.1016/j.nima.2016.03.005.

[38] G. Stancari, A. Valishev, G. Annala, G. Kuznetsov, V. Shiltsev, D. Still, L. Vorobiev, Physical Review Letters 107 (8) (2011) 084802.

[39] P. Craievich, S. Di Mitri, M. Milloch, G. Penco, F. Rossi, Physical Review Special Topics-Accelerators and Beams 16 (9) (2013) 090401.

[40] B. Marchetti, A. Bacci, E. Chiadroni, A. Cianchi, M. Ferrario, A. Mostacci, R. Pompili, C. Ronsivalle, B. Spataro, I. Zagorodnov, Review of Scientific Instruments 86 (7) (2015) 073301. 